Mon. Not. R. Astron. Soc. 000,1-?? (2005) Printed 22 October $2018 \quad$ (MN LATEX style file v2.2)

\title{
Formation of massive globular clusters with heavy element abundance spread in the Galactic building blocks
}

\author{
Kenji Bekki ${ }^{1 \star}$ \\ ${ }^{1}$ ICRAR M468 The University of Western Australia 35 Stirling Hwy, Crawley Western Australia, 6009
}

Accepted, Received 2005 February 20; in original form

\begin{abstract}
A growing number of recent observations have revealed that the Galactic globular cluster (GC) $\omega$ Cen is not the only GC that shows abundance spread in heavy elements (e.g., Fe). In order to understand the origin of the Galactic GCs with heavy element abundance spread ("HEAS"), we investigate the formation processes of massive GCs (MGCs) with masses larger than $10^{6} \mathrm{M}_{\odot}$ in gas-rich dwarf galaxies interacting and merging with the very young Galaxy. We find that massive and compact stellar clumps with masses larger than $10^{6} \mathrm{M}_{\odot}$, which can be regarded as progenitors of MGCs, can form from massive gas clumps that are developed through merging of gaseous regions initially at different radii and thus with different metallicities. Therefore it is inevitable that MGCs formed in dwarfs have HEAS. The abundance spread in each individual MGC depends on the radial metallicity gradient of the host dwarf such that it can be larger for the steeper metallicity gradient. For example, MGCs formed in a dwarf with a central metallicity of $[\mathrm{Fe} / \mathrm{H}]=-1.1$ and the radial gradient of $\sim-0.2 \mathrm{dex} \mathrm{kpc}^{-1}$ can have the abundance spread of $\Delta[\mathrm{Fe} / \mathrm{H}] \sim 0.2$. The simulated MGCs appear to be significantly flattened owing to their dissipative formation from gas disks of their host dwarfs. Based on these results, we discuss possibly diverse formation mechanisms for the Galactic GCs such as M22, M54, NGC 2419, $\omega$ Cen, and Terzan 5.
\end{abstract}

Key words: globular cluster: general - galaxies: star clusters: general - galaxies: stellar content - stars:formation

\section{INTRODUCTION}

One of remarkable recent developments in observational studies of the Galactic GCs is that most of the investigated GCs show varying degrees of chemical abundance spread: helium abundance spread in $\omega$ Cen and NGC 2808 (e.g., Bedin et al. 2004; Piotto et al. 2007), a larger dispersion in $s$-process elements abundances in NGC 1851 (e.g., Yong \& Grundahl 2008; Milone et al. 2011), abundance spread in light element in "normal" GCs (e.g., Carretta et al. 2010a), and HEAS in M22, Terzan 5, and NGC 2419 (e.g., Da Costa et al. 2009; Ferraro et al. 2009; Marino et al. 2009; Cohen et al. 2010). Lee et al. (2009) investigated color-magnitude diagrams of stars in $h k$-bands for the Galactic GCs and suggested that a significant fraction of the Galactic GCs have two different populations with HEAS (but see also Marino et al. 2009; Carretta et al. 2010b for similar and different claims).

The origin of the HEAS in $\omega$ Cen has been discussed by theoretical models in the context of formation and evolution of stellar galactic nuclei in dwarfs ("the stripped nucleus sce-

\footnotetext{
* E-mail: bekki@cyllene.uwa.edu.au
}

nario"; e.g., Bekki \& Freeman 2003; Romano et al. 2010). Since stellar populations of stellar galactic nuclei can have different metallicities and ages owing to their possible formation by merging of different stellar and gaseous clumps (e.g., Bekki 2007), it would not be so surprising that $\omega$ Cen is observed to have HEAS owing to its possible origin from the stellar nucleus of its host dwarf. Also, it would be possible that the formation process of other GCs with HEAS (e.g., Terzan 5) can be different from that of $\omega$ Cen (e.g., GC mergers; Brüns \& Kroupa 2011; Bekki \& Yong 2011, BY11). Ideally speaking, chemodynamical simulations of GC formation based on a theoretical model are essential for discussing both the formation processes of GCs from gas and the resultant internal chemical abundance spread within GCs in a fully self-consistent manner. However no chemodynamical simulations on the origin of GCs with HEAS (other than $\omega$ Cen) have been done so far.

The purpose of this Letter is to clearly show whether and how GCs with HEAS can be formed by using chemodynamical simulations of GC formation processes based on a GC formation scenario. We here consider that most of the Galactic GCs were formed in galactic building blocks (e.g., Searle \& Zinn 1978), in particular, when they were inter- 
acting and merging with the very young Galaxy ( $>10$ Gyr ago). We focus exclusively on $[\mathrm{Fe} / \mathrm{H}] \operatorname{spread}(\Delta[\mathrm{Fe} / \mathrm{H}])$ in MGCs with masses as large as or larger than $10^{6} \mathrm{M}_{\odot}$ formed within their host dwarfs (i.e., galactic building blocks).

\section{THE MODEL}

We numerically investigate star formation and chemical evolution processes of a gas-rich dwarf interacting and merging with the Galaxy in order to investigate whether MGCs composed of many stellar particles can be formed. In order to simulate chemodynamical evolution of the gas-rich dwarf in the young Galaxy, we use both the latest version of GRAPE (GRavity PipE, GRAPE-DR), which is the special-purpose computer for gravitational dynamics (Sugimoto et al. 1990), and high-end PCs with GPU cards (GTX 580) and CUDA G5/G6 software package being installed for calculations of gravitational dynamics. We adopt our original GRAPE-SPH code (Bekki 2009) which combines the method of smoothed particle hydrodynamics (SPH) with GRAPE for calculations of three-dimensional self-gravitating fluids in astrophysics.

The dwarf galaxy is assumed to be strongly influenced by the fixed gravitational potential of the Galaxy in the preset study. We adopt a model of the Galaxy similar to that used in BY11 in which the Galaxy is assumed have three components: a dark matter halo, a disk, and a bulge. We consider that (i) dwarfs forming and hosting GCs interact or merge with the Galaxy in the very early history of the Galaxy formation (>10 Gyr ago) and thus (ii) the Galaxy has not yet fully developed its halo, disk, and bulge components at the epoch of dwarf merging.

We assume the following logarithmic dark matter halo potential for the Galaxy,

$\Phi_{\text {halo }}=v_{\text {halo }}^{2} \ln \left(r^{2}+d^{2}\right)$,

where $d=12 \mathrm{kpc}, v_{\text {halo }}=131.5 \mathrm{~km} \mathrm{~s}^{-1}$ and $r$ is the distance from the center of the Galaxy. The gravitational potential of the Galactic disk is represented by a Miyamoto-Nagai (1975) potential;

$\Phi_{\mathrm{disk}}=-\frac{G M_{\mathrm{disk}}}{\sqrt{R^{2}+\left(a+\sqrt{z^{2}+b^{2}}\right)^{2}}}$,

where $M_{\text {disk }}=1.0 \times 10^{10} M_{\odot}$, and $a=6.5 \mathrm{kpc}, b=0.26$ kpc, and $R=\sqrt{x^{2}+y^{2}}$. The disk mass is much smaller than the one adopted in BY11, because we consider that only the first generation of the thin disk is being built when the dwarf starts interacting/merging with the Galaxy $(>10$ Gyr ago). We do not include the bulge in the young Galaxy, because we consider that the bulge can be formed much later from bar instability of disk stars.

The center of the Galaxy is always set to be $(x, y, z)=$ $(0,0,0)$ whereas the initial location and velocity of the dwarf are free parameters that can control the orbital evolution of the dwarf. The initial distance of the dwarf from the Galactic center and the velocity are represented by $R_{\mathrm{i}}$ and $f_{\mathrm{v}} v_{\mathrm{c}}$, respectively, where $v_{\mathrm{c}}$ is the circular velocity at $R_{\mathrm{i}}$. The inclination angle between the initial orbital plane and the $x-z$ plane (=Galactic disk plane) is denoted as $\theta$. The initial spin of the dwarf disk is specified by two angles, $\theta_{\mathrm{d}}$ and $\phi_{\mathrm{d}}$, where $\theta$ is the angle between the $z$-axis and the vector of the
Table 1. Description of the model parameters for the representative models.

$\begin{array}{crrrrc}\text { Model no. } a & M_{\mathrm{s}, \mathrm{dw}} \underline{b} & R_{\mathrm{s}, \mathrm{dw}} \underline{c} & f_{\mathrm{g}} \frac{d}{2} & \alpha_{\mathrm{d}} \frac{e}{2} & y_{\mathrm{met}} f \\ \text { M1 } & 1.0 & 1.8 & 0.5 & -0.2 & - \\ \text { M2 } & 1.0 & 1.8 & 0.5 & -0.1 & - \\ \text { M3 } & 1.0 & 1.8 & 0.5 & -0.05 & - \\ \text { M4 } & 1.0 & 1.8 & 0.1 & -0.2 & - \\ \text { M5 } & 1.0 & 1.8 & 0.2 & -0.2 & - \\ \text { M6 } & 1.0 & 2.8 & 0.5 & -0.2 & - \\ \text { M7 } & 1.0 & 1.8 & 0.5 & -0.2 & 0.0005 \\ \text { M8 } & 1.0 & 1.8 & 0.5 & -0.2 & 0.002 \\ \text { H1 } & 1.0 & 1.8 & 0.5 & -0.2 & -\end{array}$

$a$ Only the model H1 is the higher resolution model with $N=10^{6}$.

$b$ The total mass of a stellar disk in a dwarf in units of $10^{8} \mathrm{M}_{\odot}$.

$c$ The initial size of a stellar disk in a dwarf in units of kpc.

$d$ The initial gas mass fraction $\left(M_{\mathrm{g}, \mathrm{dw}} / M_{\mathrm{s}, \mathrm{dw}}\right)$.

$e$ The radial metallicity gradient of the gas disk in a dwarf (dex $\left.\mathrm{kpc}^{-1}\right)$.

$f$ The value of a chemical yield ( $y_{\text {met }}$ ) is not shown for models without chemical evolution.

angular momentum of a disk and $\phi$ is the azimuthal angle measured from $x$-axis to the projection of the angular momentum vector of a disk onto the $x-y$ plane. In the present study, we show the results of the models with $R_{\mathrm{i}}=35 \mathrm{kpc}$, $f_{\mathrm{v}}=0.5, \theta=30^{\circ}, \theta_{\mathrm{d}}=15^{\circ}$, and $\phi_{\mathrm{d}}=45^{\circ}$.

We adopt the same dwarf galaxy model as that in BY11: the dwarf has a "cored" dark matter halo (Salucci \& Burkert 2000) and an exponential stellar disk. We describe the result of the models with the total stellar disk masses $\left(M_{\mathrm{s}, \mathrm{dw}}\right)$ being $10^{8} \mathrm{M}$ in the present study: other models with different $M_{\mathrm{s}, \mathrm{dw}}$ will be discussed in our forthcoming papers. The mass-ratio of dark matter halo to stellar disk in the dwarf is set to be 9 and thus the core radius $\left(a_{\mathrm{dm}}\right)$ of the dark matter halo is $1.5 \mathrm{kpc}$. The stellar disk has the total mass of $M_{\mathrm{s}, \mathrm{dw}}$ and the size of $R_{\mathrm{s}, \mathrm{dw}}$. and the radial $(R)$ and vertical $(Z)$ density profiles of the stellar disk are assumed to be proportional to $\exp \left(-R / R_{0}\right)$ with scale length $R_{0}=0.2 R_{\mathrm{s}, \mathrm{dw}}$ and to $\operatorname{sech}^{2}\left(Z / Z_{0}\right)$ with vertical scale height $Z_{0}=0.04 R_{\mathrm{s}, \mathrm{dw}}$, respectively. In addition to the rotational velocity caused by the gravitational field of disk and dark halo components, the initial radial and azimuthal velocity dispersions are assigned to the disc component according to the epicyclic theory with Toomre's parameter $Q=1.5$. The vertical velocity dispersion at a given radius is set to be 0.5 times as large as the radial velocity dispersion at that point.

In the present study, the dwarf also has an extended gas disk with the total mass of $M_{\mathrm{g}, \mathrm{dw}}$ and the size of $R_{\mathrm{g}, \mathrm{dw}}$ $\left(=2.5 R_{\mathrm{s}, \mathrm{dw}}\right)$. The disk is assumed to have an exponential profile with scale length $0.4 R_{\mathrm{g}, \mathrm{dw}}$ and vertical scale height $0.04 R_{\mathrm{g}, \mathrm{dw}}$. The mass-ratio of gas disk to stellar one $\left(f_{\mathrm{g}}\right)$ is an important free parameter that can control the formation processes of MGCs in the present study. We consider that the dwarf disk initially has a plenty of cold gas and accordingly an isothermal equation of state is adopted for the gas with a temperature $\left(T_{\mathrm{g}}\right)$ of $160 \mathrm{~K}$. Star formation is modeled by converting the collisional gas particles into collisionless new stellar particles according to the algorithm of star formation described below. We adopt the Schmidt law with exponent $\gamma=1.5(1.0<\gamma<2.0$, Kennicutt 1998) as the controlling parameter of the rate of star formation. 

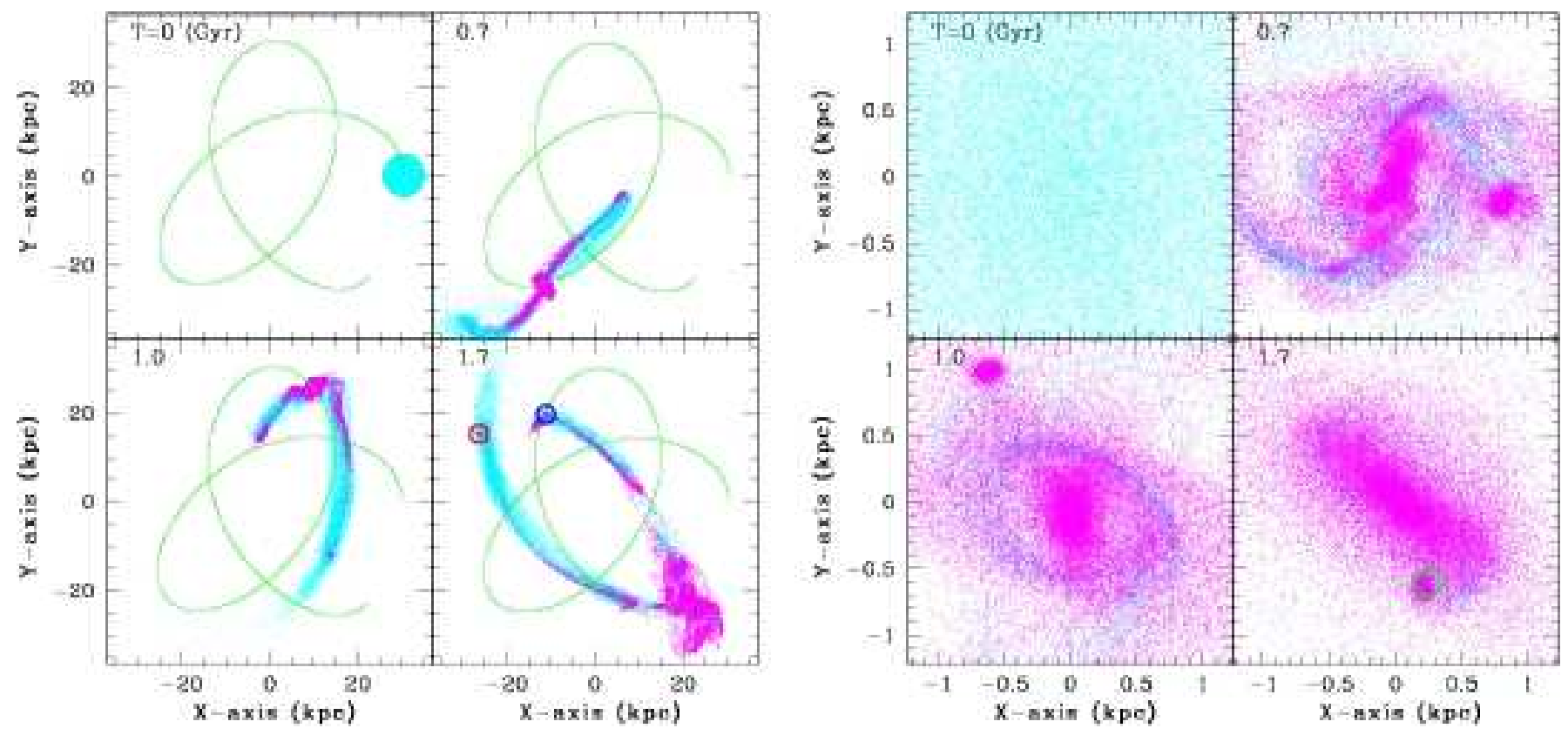

Figure 1. The time evolution of the mass distribution of the dwarf with respect to the Galactic center (left four) and to the dwarf's center (right four) projected onto the $x-y$ plane for the standard model (M1). Gas and new stars are shown by cyan and magenta, respectively, and $T$, which is the time that has elapsed since the simulation started, is shown in the upper left corner in units of Gyr for each panel. The green lines in the left four panels represent the orbit of the dwarf. The locations of MGC1, 2, and 3 are indicated by blue, red, and green circles at $T=1.7 \mathrm{Gyr}$, respectively.

We allocate metallicity to each gas particle according to its initial position: at $r=R$, where $r(R)$ is the projected distance (in units of kpc) from the center of the disk, and thus the metallicity of the gas is given as:

$[\mathrm{Fe} / \mathrm{H}]_{\mathrm{r}=\mathrm{R}}=[\mathrm{Fe} / \mathrm{H}]_{\mathrm{d}, \mathrm{r}=0}+\alpha_{\mathrm{d}} \times \mathrm{R}$.

We consider that (i) the slope $\alpha_{\mathrm{d}}$ is a free parameter and (ii) $[\mathrm{Fe} / \mathrm{H}]_{\mathrm{d}, \mathrm{r}=0}$ is determined by the total stellar mass of the dwarf. Accordingly we adopt $[\mathrm{Fe} / \mathrm{H}]=-1.1$ as a reasonable value for the adopted $M_{\mathrm{s}, \mathrm{dw}}$, and the observed value of $\alpha_{\mathrm{d}} \sim-0.2$ for dwarf spirals (Hidalgo-Gámez et al. 2010) should be a reference value for the radial metallicity gradient. The chemical yield $y_{\text {met }}$ and the return parameter $R_{\text {met }}$ are key parameters that control chemical evolution of dwarfs. We investigate models with (i.e., $y_{\text {met }}>0$ and a fixed $\left.R_{\text {met }}=0.3\right)$ and without $\left(y_{\text {met }}=0\right)$ chemical enrichment by supernovae.

In order to find massive stellar clumps with masses $\left(m_{\mathrm{ns}}\right)$ as large as or larger than $10^{6} \mathrm{M}_{\odot}$, we investigate the total number of new stellar particles $\left(N_{\text {nei }}\right)$ within $200 \mathrm{pc}$ around each new stellar particle. If $N_{\text {nei }}$ around a new stellar particle is larger than a threshold value $n_{\text {thres }}$, then we regard the particle as being within a massive and compact stellar clump. Since the particle is not necessarily at the center of the clump, we newly determine the center of the clump using the position data of the neighboring particles around the particle. For the newly determined center of the clump, we estimate the total mass of the clump within the central 200pc. We consider that $n_{\text {thres }}=1000$ is reasonable, firstly because only massive clumps $\left(m_{\mathrm{ns}} \geqslant 5 \times 10^{5} \mathrm{M}_{\odot}\right.$ for $\left.f_{\mathrm{g}}=0.5\right)$ can be selected and secondly because the selected clumps also show high stellar densities.

The selected massive clumps are significantly larger and more massive than the present GCs and thus should be regarded as progenitors of GCs (i.e., not literally genuine GCs). Recent numerical studies of multiple stellar populations in GCs have suggested that the original GC should be much larger and more massive than the present ones (e.g., D'Ercole et al. 2008; Bekki 2011). Therefore, the adopted assumption that massive stellar clumps can be progenitors of GCs is reasonable and realistic. The final massive stellar clumps are referred to as MGCs for convenience in the present study. Owing to the introduction of a gravitational softening length for new stars, the present simulations are not suitable for investigating the long-term internal dynamical evolution of GCs. We will discuss the long-term dynamical evolution processes of GCs after their formation in gas-rich dwarfs in our forthcoming paper by using $\mathrm{N}$-body codes suitable for GC internal dynamics. Table 1 summarizes nine representative models for which the results are discussed in the present paper. We mainly describe the results of the "standard" model (M1) in which $f_{\mathrm{g}}=0.5$, $\alpha_{\mathrm{d}}=-0.2$, and chemical enrichment is not included (i.e., $\left.y_{\text {met }}=0\right)$. This is mainly because we need to more clearly demonstrate the origin of HEAS in GCs. The total particle numbers for the dark matter $\left(N_{\mathrm{dm}}\right)$, the stellar disk $\left(N_{\mathrm{s}}\right)$, and the gas one $\left(N_{\mathrm{g}}\right)$ used in most of the present models are 200,000, 200,000, 100,000, respectively. The gravitational softening lengths $(\epsilon)$ for the dark matter particles, the stellar ones, and the gaseous ones are 128pc, 20pc, and 20pc, respectively. In order to confirm that the present results do not depend on the adopted numerical resolution, we also run a high-resolution model (H1) in which $N_{\mathrm{dm}}=400,000$ $N_{\mathrm{s}}=400,000$ and $N_{\mathrm{g}}=200,000$. 

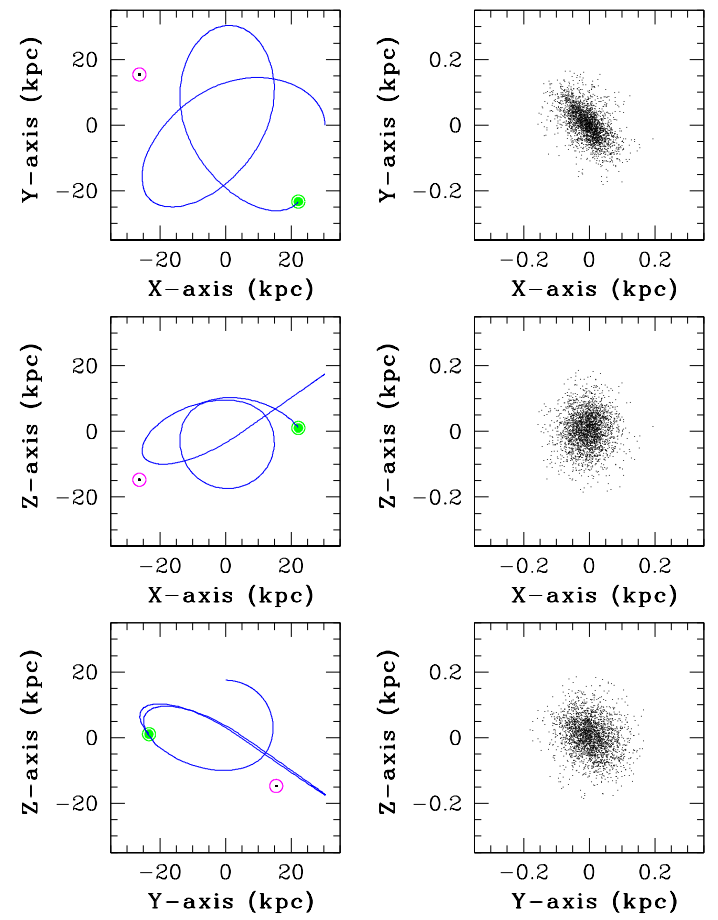

Figure 2. The orbits of the dwarf (left three) and the mass distributions of MGC2 (right three) projected onto the $x-y$ (top), $x-z$ (middle), and $y-z$ planes (bottom) in the standard model (M1). For comparison, the locations of the dwarf and MGC2 at $T=1.7 \mathrm{Gyr}$ are shown by green and magenta circles, respectively. Only new stars formed from gas are plotted in the right three panels.
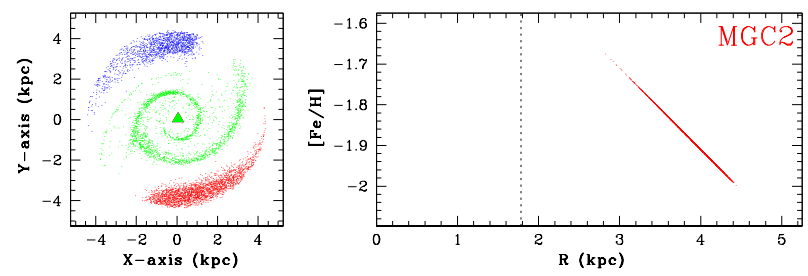

Figure 3. The original location of new stars of MGC1 (blue), 2 (red), and 3 (green) within the initial dwarf disk $(T=0$ Gyr) projected onto the $x$-y plane (left) and the initial locations of original gas particles for new stars of MGC2 on the $R$ - $[\mathrm{Fe} / \mathrm{H}]$ plane (right). The green triangle represents the final location of MGC3 within the host at $T=3.4$ Gyr (i.e., evolution into nucleus). The vertical dotted line in the right panel represents the size of the stellar disk of the host dwarf $\left(R_{\mathrm{S}, \mathrm{dw}}\right)$.

\section{RESULTS}

Fig. 1 shows the orbital evolution of the dwarf and the time evolution of the mass distributions of gas and new stars in the standard model (M1). During the first pericenter passage $\left(R_{\mathrm{p}} \sim 14 \mathrm{kpc}\right)$ at $T \sim 0.3 \mathrm{Gyr}$, the gas disk is strongly compressed by the tidal field of the Galaxy to form highdensity gaseous regions. As a result of this, a number of massive clumps composed mostly of gas and new stars can be formed within the central $\sim 1 \mathrm{kpc}$ of the dwarf and the star formation rate becomes higher $\left(\sim 0.08 \mathrm{M}_{\odot} \mathrm{yr}^{-1}\right)$. After the pericenter passage, a significant fraction of gas and new stars are tidally stripped and the stripped matter can form

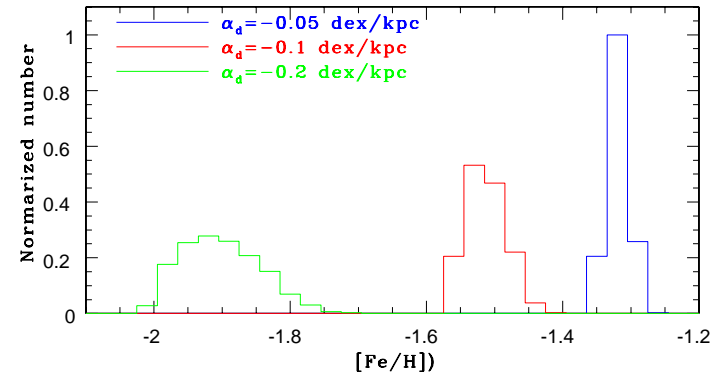

Figure 4. The $[\mathrm{Fe} / \mathrm{H}]$ distributions of new stars in MGC2 for the models M3 with $\alpha_{\mathrm{d}}=-0.05$ (blue), M2 with $\alpha_{\mathrm{d}}=-0.1$ (red), and M1 (i.e., the standard model) with $\alpha_{\mathrm{d}}=-0.2$ (green).

leading and trailing streams along the orbit of the dwarf $(T=0.7$ Gyr $)$. Some of the more massive clumps can sink into the central region of the dwarf owing to dynamical friction so that they can become stellar galactic nucleus. At the final time step ( $T=1.7 \mathrm{Gyr}$ ), only one massive and compact clump (identified as MGC3) with $m_{\mathrm{ns}}=1.2 \times 10^{6} \mathrm{M}_{\odot}$ (originally $1.6 \times 10^{6} \mathrm{M}_{\odot}$ at $T=1.0 \mathrm{Gyr}$ ) can be clearly seen within the central $1 \mathrm{kpc}$ of the dwarf. We confirm that this MGC3 can sink into the dwarf's center within the next $\sim 1$ Gyr to become nucleus: it can be identified as an isolated GC only after the complete destruction of its host by the (older) Galaxy with the much larger mass.

Two stellar clumps can be developed from gaseous tidal tails (or outer spiral arms) during the tidal interaction of the dwarf with the Galaxy. Fig. 1 shows that the two clumps (i.e., MGC progenitors) can be stripped from their host dwarf and consequently located within the gaseous tails $(T=1.7 \mathrm{Gyr})$. These two clumps are identified as MGC1 with $m_{\mathrm{ns}}=7.5 \times 10^{5} \mathrm{M}_{\odot}$ and $\mathrm{MGC} 2$ with $m_{\mathrm{ns}}=1.3 \times 10^{6} \mathrm{M}_{\odot}$. They can be finally regarded as "isolated" GCs owing to their large distances (> $40 \mathrm{kpc}$ ) from the dwarf. Fig. 2 shows that owing to its formation from the outer gas disk through gaseous dissipation, MGC2 has a significantly flattened shape in the $x-y$ projection. This flattened shape can be seen in most of the simulated MGCs in the present study.

The formation of MGCs can be severely suppressed in models (M4 and 5) with lower $f_{\mathrm{g}}(\leqslant 0.2)$, which implies that MGC formation is possible only when their host dwarfs have a plenty of cold gas. Therefore the formation processes of MGCs in gas-rich disks and tidal tails described above can be essentially the same as those of massive stellar and gaseous clumps discussed in previous papers (e.g., Noguchi 1999; e.g., Bournaud et al. 2007) in which $f_{\mathrm{g}}$ is a fundamentally important parameter for dynamical evolution of gas-rich galactic disks. MGCs can be formed even in the low surface brightness model M6 with larger $R_{\mathrm{s}, \mathrm{dw}}(=2.8 \mathrm{kpc})$, which implies that mass densities of stellar disks in dwarfs are not so important as $f_{\mathrm{g}}$ for MGC formation.

Fig. 3 clearly shows that new stars of MGC2 originate from gaseous regions at different locations within the dwarf and thus with different $[\mathrm{Fe} / \mathrm{H}]$ owing to the adopted metallicity gradient of the gas disk. This result demonstrates that some Galactic GCs have HEAS because they formed from massive gaseous clumps developed from merging of different gaseous regions with different initial $[\mathrm{Fe} / \mathrm{H}]$. However, Fig. 4 shows that the degree of $\operatorname{HEAS}(\Delta[\mathrm{Fe} / \mathrm{H}])$ depends strongly 


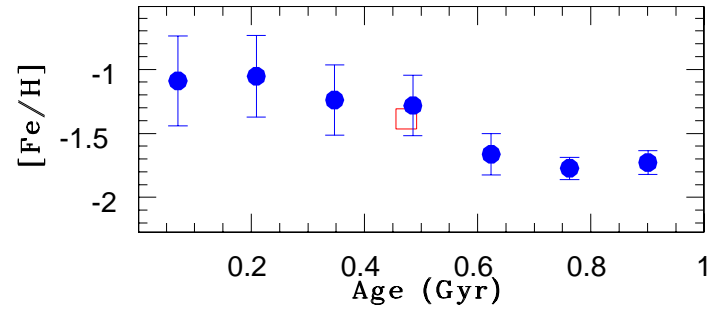

Figure 5. The age-metallicity relation for MGC2 in the model M7 with chemical evolution $\left(y_{\text {met }}=0.0005\right)$. Here "ages" $\left(t_{\text {age }}\right)$ of stars are estimated at $T=1.7 \mathrm{Gyr}$ (i.e., the final time step of the simulation): $t_{\text {age }}=0$ means star formation at $T=1.7$ Gyr. The mean value and dispersion of $[\mathrm{Fe} / \mathrm{H}]$ at each age bin are shown by a filled blue circle and an error bar, respectively. The red square represents the mean age and metallicity of MGC2.

on $\alpha_{\mathrm{d}}$ such that $\Delta[\mathrm{Fe} / \mathrm{H}]$ is larger $(\sim 0.2 \mathrm{dex})$ for the steeper metallicity gradient (i.e., the larger absolute magnitude of $\left.\alpha_{\mathrm{d}}\right)$.

Fig. 5 shows that a younger population can have $[\mathrm{Fe} / \mathrm{H}] \sim-1$ in $\mathrm{MGC} 2$ in the model with chemical evolution (M7), though the mass fraction the population with the age less than 0.2 Gyr is only $\sim 0.05$. Although the prolonged star formation can be seen, the vast majority of stars are formed in a burst at the epoch of massive clump formation. Furthermore, there appears to be an age-metallicity relation that younger stars are more metal-rich in MGC2. It should be stressed that the relation can depend on how the supernova ejecta can influence the later star formation within cluster-forming massive gas clumps, which needs to be investigated by more sophisticated models in our future works: The $[\mathrm{Fe} / \mathrm{H}]$ spread could be overestimated owing to the adopted instantaneous recycling approximation. The mean metallicities and internal $[\mathrm{Fe} / \mathrm{H}]$ spread of MGCs are larger in models with larger $y_{\text {met }}$ (e.g., M8) in the present study. The physical properties of MGCs (e.g., masses and locations) are not different between models with different numerical resolutions (M1 and $\mathrm{H} 1$ ).

\section{DISCUSSION AND CONCLUSIONS}

The present chemodynamical study has first demonstrated that MGCs with HEAS can be formed from massive gas clumps developed from merging of different gaseous regions with different metallicities. Therefore, MGCs with HEAS can be formed even without chemical enrichment by supernovae during their formation. The present study suggests that more massive GCs are more likely to have a larger degree of HEAS owing to their formation from gas clouds developed from merging between a larger number of different gaseous regions with different metallicities. The low-mass GCs can be formed from single gas clouds so that they are likely to have no/little HEAS. The present study thus suggests that the origin of HEAS in some Galactic GCs can be closely associated with HEAS of ISM in their host dwarfs.

The large metallicity spread of various elements and possible age variation in $\omega$ Cen (e.g., Sollima et al. 2005) can be consistent with the stripped nucleus scenario. However, this does not necessarily mean that all of the Galactic GCs with HEAS (e.g., NGC 2419, M22, and Terzan 5) were formed from stripped nuclei of dwarfs. A GC with distinct two peaks in the $[\mathrm{Fe} / \mathrm{H}]$ distributions of the stars might well be consistent with GC merging with different [Fe/H] (e.g., Brüns \& Kroupa 2011; BY11). A GC with a smaller degree of HEAS yet no clear bimodal $[\mathrm{Fe} / \mathrm{H}]$ distribution might well form from massive gas clumps of the host dwarf and then be stripped from the dwarf without sinking into the center (i.e., without becoming the stellar nucleus) to finally become the Galactic halo GC. We lastly suggest that nucleation, GC merging, and merging of gas clouds with different $[\mathrm{Fe} / \mathrm{H}]$ can be all promising mechanisms for the formation of the Galactic GCs with different degrees of HEAD.

\section{ACKNOWLEDGMENT}

I am grateful to the anonymous referee for constructive and useful comments that improved this paper.

\section{REFERENCES}

Bedin, L. R., Piotto, G., Anderson, J., Cassisi, S., King, I. R., Momany, Y., Carraro, G., 2004, ApJ, 605, L125

Bekki, K., 2007, PASA, 24, 77

Bekki, K. 2009, MNRAS, 399, 2221

Bekki, K., 2011, MNRAS, 412, 2241

Bekki, K., Freeman, K. C., 2003, MNRAS, 346, L11

Bekki, K., Yong, D., 2011, MNRAS in press (arXiv1109.4463)

Bournaud, F., Elmegreen, B. G., Elmegreen, D. M., 2007, ApJ, 670, 1194

Brüns, R. C., Kroupa, P., 2011, ApJ, 729, 69

Carretta, E., et al. 2010, A\&A, 516, 55 (2010a)

Carretta, E., et al., 2010, ApJ, 722, L1 (2010b)

Cohen, J. G., et al., 2010, ApJ, 725, 288

Da Costa, G. S., Held, E. V., Saviane, I., Gullieuszik, M., 2009, ApJ, 705, 1481

D'Ercole, A., Vesperini, E., D'Antona, F., McMillan, S. L. W., \& Recchi, S. 2008, MNRAS, 391, 825

Ferraro, F. R., et al. 2009, Nature, 462, 483

Hidalgo-Gamez, A. M., Ramirez-Fuentes, D., Gonzalez, J. J., 2010, preprint (arXiv1011.1013)

Kennicutt, R. C. 1989, ApJ, 344, 685

Lee, J-W., Kang, Y-W., Lee, J., \& Lee, Y-W. 2009, Nature, 462,480

Marino, A. F., et al. 2009, A\&A, 505, 1099

Milone, A. P., et al., 2009, A\&A, 497, 755

Milone, A. P., et al., 2011, preprint (arXiv1107.2056)

Miyamoto, M., Nagai, R., 1975, PASJ, 27, 533

Noguchi, M., 1999, ApH, 514, 77

Piotto, G. et al. 2007, ApJ, 661, L53

Romano, D., et al., 2010, MNRAS, 401, 2490

Salucci, P., Burkert, A., 2000, ApJL, 537, 9

Searle, L. \& Zinn, R., 1978, ApJ, 225, 357

Sollima, A., Pancino, E., Ferraro, F. R., Bellazzini, M., Straniero, O., Pasquini, L., 2005, ApJ, 634, 332

Sugimoto, D., et al., 1990, Nature, 345, 33

Yong, D., Grundahl, F., 2008, ApJ, 672, 29 